\title{
Diffusion quasiélastique de neutrons ; étude des mouvements localisés
}

\author{
P. Damay et F. Leclercq
}

LASIR, UMR 8516 du CNRS, HEI, 13 rue de Toul, 59046 Lille cedex, France

\begin{abstract}
La localisation des mouvements (sauts sur un nombre fini de sites, rotation, diffusion dans un volume fini) induit un effet de mémoire caractérisé par $\langle\mathrm{I}(\mathrm{Q}, \mathrm{t}=0) \bullet \mathrm{I}(\mathrm{Q}, \mathrm{t}=\infty)\rangle \neq 0$. Lintensité diffusée, proportionnelle au facteur de structure dynamique incohérent $S(Q, \omega)$ se présente sous la forme d'un pic quasiélastique globalement lorentzien centré à $\omega=0$, surmonté d'un pic élastique qui renseigne sur la localisation du mouvement (EISF).

Quelques exemples simples (sauts entre deux sites équivalents, deux sites non-équivalents..) sont expliqués en détail et résolus manuellement.

La généralisation de la méthode, basée sur la résolution matricielle, permet d'aborder des systèmes plus complexes (plusieurs temps caractéristiques et plusieurs distances de saut). La réorientation dans les cristaux moléculaires est abordée en utilisant la théorie des groupes. Quelques exemples originaux sont traités en détail et les résultats sont comparés à ceux obtenus par la résolution matricielle.
\end{abstract}

\section{INTRODUCTION}

Dans les cristaux moléculaires, les molécules sont attachées à des sites cristallographiques, et, aux faibles échanges d'énergie, la dynamique est restreinte à des mouvements de réorientation autour des axes de symétrie. Aux très basses températures, la rotation est empêchée par des barrières de potentiel créées par le champ cristallin et les seuls mouvements possibles sont de type quantique. L'énergie des pics de rotation par effet tunnel dépend du moment d'inertie (via $\hbar / 2 I$ ) et décroît exponentiellement avec la hauteur de la barrière. Pour un groupement $-\mathrm{CH}_{3}$, par exemple, l'énergie des pics observés peut aller du $\mu \mathrm{eV}$, dans le cas de barrières élevées, jusqu'à la rotation libre à $0.7 \mathrm{meV}$. Une élévation de température a pour effet de superposer un potentiel fluctuant dépendent du temps $V(t)$ au potentiel statique. Si les potentiels créés par le champ cristallin restent importants devant l'excitation thermique, les mouvements seront restreints à des sauts entre les sites cristallographiques; par contre si l'excitation devient beaucoup plus importante que les potentiels locaux, on pourra observer des mouvements de diffusion continus autour d'un axe de symétrie, voire autour du centre de symétrie. Ces deux types de mouvement classiques produisent un spectre de diffusion quasiélastique, c'est-à-dire une distribution d'échanges d'énergie centrée sur le pic élastique. L'étude pratique de la dynamique réorientationnelle peut ainsi, comme dans le cas des liquides, être abordée à partir des deux approches limites : soit une réorientation par sauts sur des sites ponctuels lorsque le temps de résidence dans un site est beaucoup 
plus long que le temps passé à effectuer le saut, soit une réorientation suivant une loi de diffusion rotationnelle (loi de Fick), la réalité physique faisant souvent appel à un mécanisme hybride.

Dans le cas de mouvements localisés, le facteur de structure incohérent $S(Q, \omega)$ est toujours la somme d'une composante élastique $A_{o}(Q) \delta(\omega)$ (Elastic Incoherent Structure Factor EISF), dont la largeur effective est la résolution du spectromètre et d'un massif quasiélastique formé de une ou plusieurs lorentziennes centrées sur l'échange d'énergie nulle. Pour chaque valeur de $Q$, on vérifie que l'intégrale $\int S(\mathbf{Q}, \omega) d \omega$ est égale à 1 , indiquant que l'intensité incohérente ne dépend pas du vecteur de transfert.

L'objectif de la mesure (ou du calcul) est de déterminer la fonction intermédiaire de diffusion incohérente $\quad I_{\text {inc }}(\mathbf{Q}, t)=\left\langle e^{i \mathbf{Q} \cdot \mathbf{r}(t)} \cdot e^{-i \mathbf{Q} . \mathbf{r}(0)}\right\rangle$ qui décrit la probabilité de trouver la particule en $r$ à $\mathrm{t}$ à partir de sa position à $\mathrm{t}=0$. Le facteur de structure incohérent $S_{\text {inc }}(Q, \omega)$ est la transformée de Fourier par rapport au temps de la fonction de diffusion intermédiaire.

Dans une première partie (Section 2), quelques exemples simples de réorientation par sauts sont décrits en détail. La méthode est ensuite généralisée et appliquée à plusieurs types de mouvements simples (Section3). L'utilisation de la théorie des groupes permet d'aborder de façon plus formelle la réorientation par rotation autour d'axes cristallographiques dans les cristaux moléculaires (Section 4). Quelques exemples de mouvements continus seront ensuite développés (Section 5). Des extensions vers des cas plus complexes sont enfin évoquées dans la dernière partie (Section 6).

\section{QUELQUES CAS SIMPLES}

\subsection{Sauts sur deux sites équivalents}

Considérons 2 sites ( $l$ et 2 ) situés aux positions $r_{1}$ et $r_{2}$. Soient $p_{l}(t)$ et $p_{2}(t)$ les probabilités de trouver la particule dans chacun des sites au temps $t$. Soit $\tau$ le temps de résidence moyen de la particule sur un site.

Comme on est sûr de trouver la particule en en 1 ou 2 :

$$
p_{1}(t)+p_{2}(t)=1
$$

L'équation de vitesse s'écrit :

$$
\begin{gathered}
\frac{d}{d t} p_{1}(t)=\frac{1}{\tau}\left[-p_{1}(t)+p_{2}(t)\right] \\
\frac{d}{d t} p_{2}(t)=-\frac{d}{d t} p_{1}(t)=\frac{1}{\tau}\left[p_{1}(t)-p_{2}(t)\right]
\end{gathered}
$$

La solution générale des équations différentielles est de la forme

$$
p(t)=1 / 2+B e^{-2 t / \tau}
$$


où la constante B est définie par les conditions initiales.

Appliquons ce résultat à la résolution de l'équation $2 a$.

Si la particule est en $r_{1}$ à $t=0, p_{1}(0)=1=1 / 2+B$ et $B=1 / 2$ et

$$
p_{11}(t)=\frac{1}{2}\left(1+e^{-2 t / \tau}\right)
$$

Si la particule est en $r_{2}$ à $t=0, p_{1}(0)=0=1 / 2+B$ et $B=-1 / 2$ et

$$
p_{21}(t)=\frac{1}{2}\left(1-e^{-2 t / \tau}\right)
$$

où $p_{I I}(t)$ est la probabilité de trouver la particule en $r_{1}$ au temps $t$ si elle était en $r_{l}$ à $t=0$ et $p_{21}(t)$ la probabilité de trouver la particule en $\boldsymbol{r}_{1}$ à $\mathrm{t}$ si elle était en $\boldsymbol{r}_{2}$ à $t=0$.

La résolution de l'équation $2 b$ décrit les deux autres événements possibles

$$
\begin{aligned}
& p_{12}(t)=\frac{1}{2}\left(1-e^{-2 t / \tau}\right) \\
& p_{22}(t)=\frac{1}{2}\left(1+e^{-2 t / \tau}\right)
\end{aligned}
$$

Les équations de vitesse décrivent le comportement d'une seule particule navigant entre les différents sites qui lui sont accessibles. Quand le système est à l'équilibre thermodynamique, le bilan des flux de toutes les particules du système s'annule, c'est-à-dire

$$
\left\langle\frac{d}{d t} p_{1}(t)\right\rangle=\frac{1}{\tau}\left[\left\langle p_{1}(t)\right\rangle-\left\langle p_{1}(t)\right\rangle\right]=\left\langle p_{1}\right\rangle-\left\langle p_{2}\right\rangle=0
$$

quel que soit $t$. Les conditions d'occupation des sites à l'équilibre thermodynamique s'obtiennent ainsi en annulant les dérivées par rapport au temps dans les équations de vitesse; dans le cas considéré $\left\langle\mathrm{p}_{1}\right\rangle=\left\langle\mathrm{p}_{2}>=1 / 2\right.$. A partir de 1,4 et 5 on obtient :

$$
I(\mathbf{Q}, t)=\left[p_{11}(t)+p_{12}(t) e^{i \cdot\left(\mathbf{r}_{2}-\mathbf{r}_{1}\right)}\right]\left\langle p_{1}\right\rangle+\left[p_{21}(t) e^{-i Q \cdot\left(\mathbf{r}_{2}-\mathbf{r}_{1}\right)}+p_{22}(t)+\right]\left\langle p_{2}\right\rangle
$$

Le premier terme rend compte de la dynamique de la particule en fonction du temps si elle était en $\boldsymbol{r}_{1}$ à $t=0$ multiplié par la probabilité de trouver la particule en $r_{l}$ à $t=0$; le second terme rend compte de la dynamique dans le cas où la particule est en $\boldsymbol{r}_{2}$ à $t=0$. Comme les $p_{i j}(\mathrm{t})$ sont des probabilités avec la somme des $p_{i j}$ égale à 1 , l'équation est bien la moyenne attendue.

La fonction intermédiaire de diffusion devient

avec

$$
I(Q, t)=A_{0}(Q)+A_{1}(Q) e^{-2 t / \tau}
$$


et

$$
A_{1}(\mathbf{Q})=\left[1-\cos \mathbf{Q} \cdot\left(\mathbf{r}_{2}-\mathbf{r}_{1}\right)\right] / 2
$$

La transformée de Fourier par rapport au temps donne le facteur de structure dynamique incohérent :

$$
S(\mathbf{Q}, \omega)=A_{0}(\mathbf{Q}) \delta(\omega)+A_{1}(\mathbf{Q}) \frac{1}{\pi} \frac{2 \tau}{4+\omega^{2} \tau^{2}}
$$

Si l'échantillon est une poudre, on doit faire la moyenne sur toutes les orientations :

$$
S(Q, \omega)=\int S(Q, \omega) d \Omega \quad \text { avec } d \Omega=\sin \theta d \theta d \varphi
$$

On obtient

$$
S(Q, \omega)=A_{0}(Q) \delta(\omega)+A_{1}(Q) \frac{1}{\pi} \frac{2 \tau}{4+\omega^{2} \tau^{2}}
$$

avec

$$
\begin{aligned}
& A_{0}(Q)=\left[1+j_{0}(Q r)\right] / 2 \\
& A_{1}(Q)=\left[1-j_{0}(Q r)\right] / 2
\end{aligned}
$$

où $j_{0}(x)$ est la fonction de Bessel sphérique d'ordre zéro est $r=\left|r_{2}-r_{1}\right|$ est la distance entre les deux sites.

Pour une poudre, on a défini le vecteur de transfert à une dimension, $Q=\frac{4 \pi}{\lambda} \sin \theta$.

Des équations 11, on vérifie que l'intégrale de $S(Q, \omega) d \omega$ est égale à $A_{0}(Q)+A_{1}(Q)=1$, indiquant que la diffusion incohérente ne dépend pas du vecteur de transfert $Q$.

L'EISF correspondant est reporté sur la figure 1. La composante quasiélastique du facteur de structure incohérent est reportée sur la figure 2.

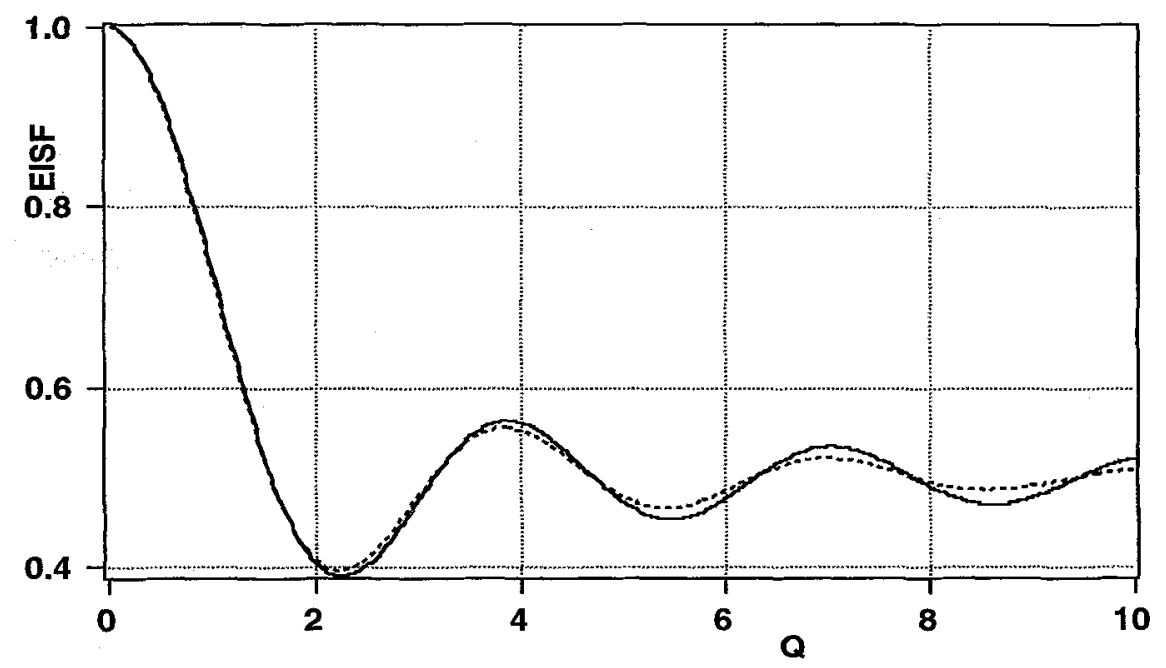

Figure 1 : EISF correspondant à la réorientation sur deux sites équivalents. La courbe en pointillé correspond au même EISF avec un faceur de Debye - Waller de $0.01 \AA^{2}$ 


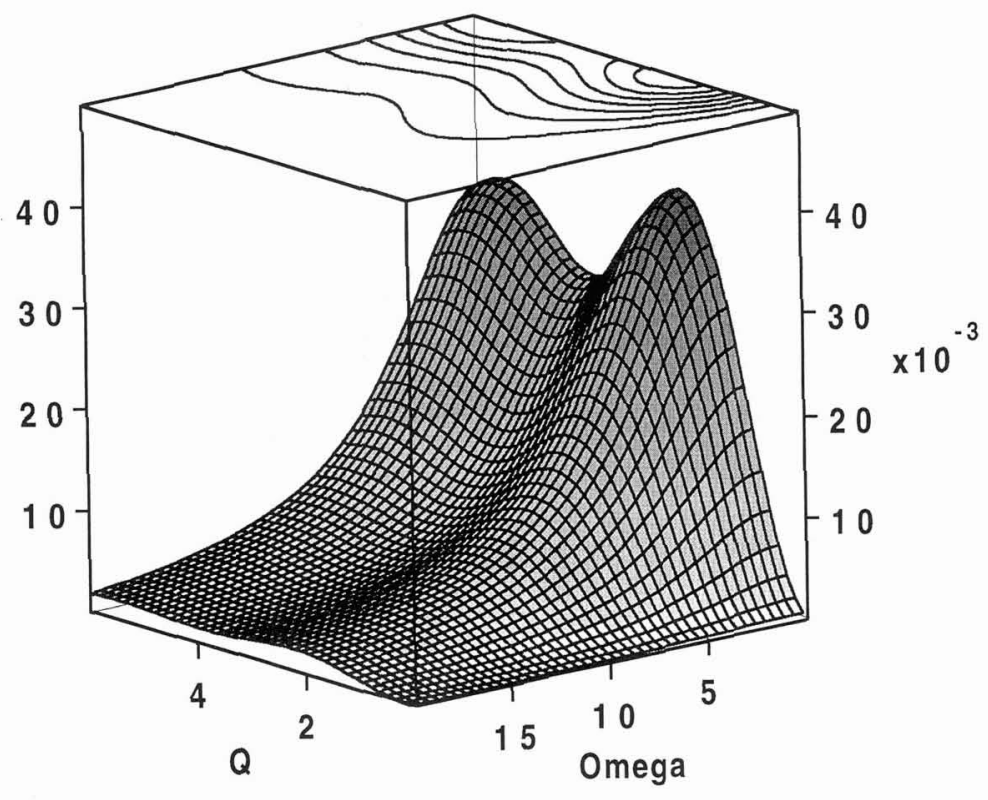

Figure 2 : Composante quasiélastique de $S(Q, \omega)$ pour une réorientation par sauts entre deux sites équivalents.

L'EISF oscille autour de 0.5, valeur qui est atteinte aux grandes valeurs de Q. La courbe en pointillé sur la figure 2 correspond au même EISF auquel a été ajouté un facteur Debye-Waller de $0.01 \AA^{2}$. Ce facteur permet de tenir compte du fait que les sites ne sont pas parfaitement ponctuels. La valeur $0.01 \AA^{2}$ correspond à $\langle\delta r\rangle=0,07 \AA$ typique d'une liaison covalente forte. L'effet du facteur Debye-Waller n'est sensible qu'aux grandes valeurs de $\mathrm{Q}\left(>3 \AA^{-1}\right)$, valeurs rarement atteintes dans les expériences de diffusion quasiélastique.

La transformée de Fourier de l'EISF est reportée sur la figure 3. On observe un pic de forme gaussienne situé à la distance $r=2 \AA$ du site 1 situé arbitrairement à l'origine. La largeur du pic est donnée par le facteur Debye-Waller. La surface sous le pic est égale à 0.5 indiquant que la probabilité de trouver la particule dans ce site est 0.5 . La surface 0.5 restante est concentrée à l'origine sur le premier site ${ }^{1}$. 


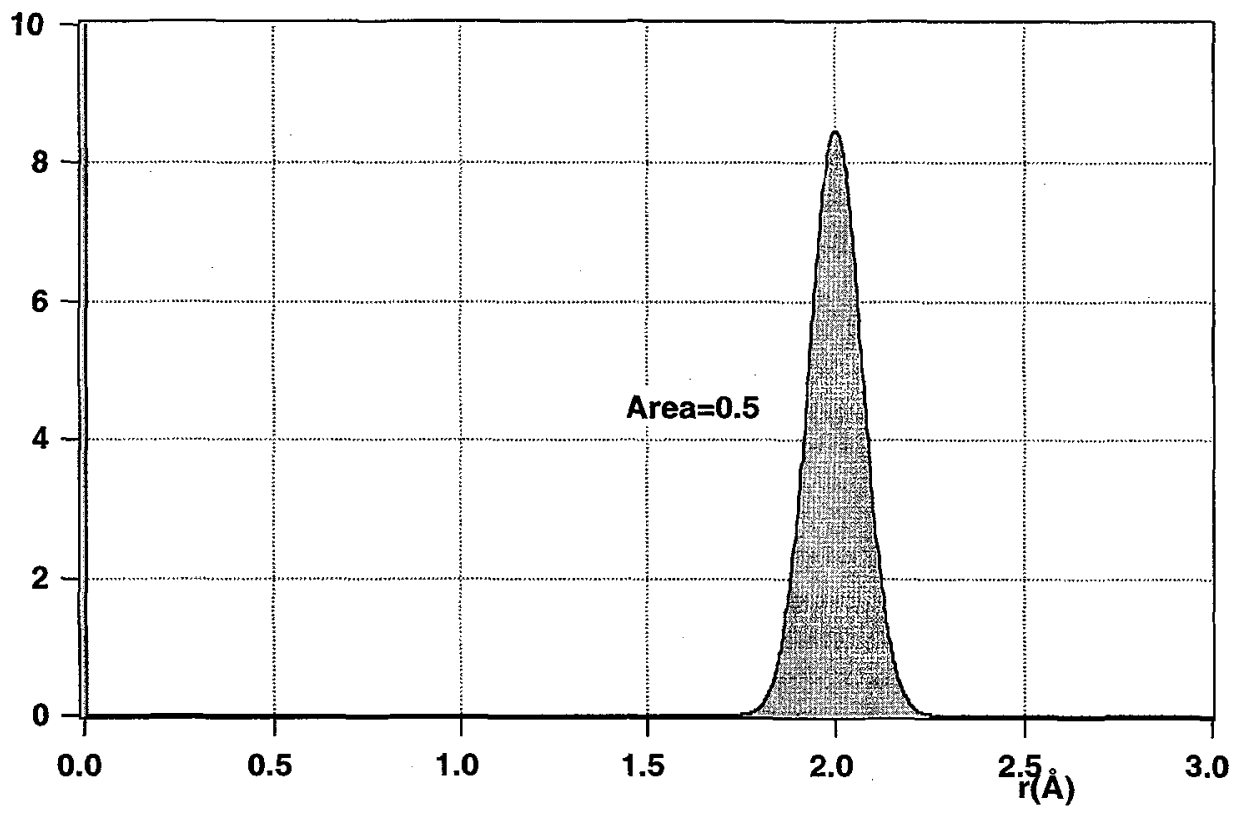

Figure 3: Transformée de Fourier de l'EISF pour la réorientation par sauts sur 2 sites distants de $2 \AA$ avec un facteur Debye-Waller de $0.01 \AA^{2}$.

\subsection{Sauts sur deux sites non-équivalents}

L'équation des vitesses s'écrit : $\quad \frac{d}{d t} p_{1}(t)=-\frac{1}{\tau_{1}} p_{1}(t)+\frac{1}{\tau_{2}} p_{2}(t)$

On conserve

$$
p_{1}(t)+p_{2}(t)=1 \text {, et } \frac{d}{d t} p_{2}(t)=-\frac{d}{d t} p_{1}(t)
$$

L'équilibre thermodynamique, indépendant du temps, est obtenu en annulant les dérivées par rapport au temps :

$$
\left\langle p_{1}\right\rangle=\frac{\tau_{1}}{\tau_{1}+\tau_{2}} \text { et }\left\langle p_{2}\right\rangle=\frac{\tau 2}{\tau_{1}+\tau_{2}}
$$


On obtient immédiatement :

$$
\begin{aligned}
& p_{11}(t)=\frac{\tau_{1}}{\tau_{1}+\tau_{2}}+\frac{\tau_{2}}{\tau_{1}+\tau_{2}} \exp \left(-t / \tau_{c}\right), \quad p_{21}(t)=\frac{\tau_{1}}{\tau_{1}+\tau_{2}}\left(1-\exp \left(-t / \tau_{c}\right)\right) \\
& p_{12}(t)=\frac{\tau_{2}}{\tau_{1}+\tau_{2}}\left(1-\exp \left(-t / \tau_{c}\right)\right), \quad p_{22}(t)=\frac{\tau_{2}}{\tau_{1}+\tau_{2}}+\frac{\tau_{1}}{\tau_{1}+\tau_{2}} \exp \left(-t / \tau_{c}\right)
\end{aligned}
$$

avec $\tau_{c}=\frac{\tau_{1} \tau_{2}}{\tau_{1}+\tau_{2}}$, le temps caractéristique.

La fonction de diffusion intermédiaire et le facteur de structure dynamique ont la même forme que dans le cas précédent :

$$
I(Q, t)=A_{0}(Q)+A_{1}(Q) \exp \left(-t / \tau_{c}\right)
$$

avec $\tau_{c}$ défini par l'équation 15 et :

$$
\begin{aligned}
& A_{0}(Q)=1-\frac{2 \tau_{c}\left[1-\cos \left(Q \cdot\left(r_{2}-r_{1}\right)\right)\right]}{\left(\tau_{1}+\tau_{2}\right)} \\
& A_{1}(Q)=\frac{2 \tau_{c}\left[1-\cos \left(Q \cdot\left(r_{2}-r_{1}\right)\right)\right]}{\left(\tau_{1}+\tau_{2}\right)}
\end{aligned}
$$

Pour une poudre, on obtient:

$$
\begin{aligned}
& A_{0}(Q)=1-\frac{2 \tau_{c}\left[1-j_{0}(Q d)\right]}{\left(\tau_{1}+\tau_{2}\right)} \\
& A_{1}(Q)=\frac{2 \tau_{c}\left[1-j_{0}(Q d)\right]}{\left(\tau_{1}+\tau_{2}\right)}
\end{aligned}
$$

Les valeurs de l'EISF pour plusieurs ratio $\tau_{l} / \tau_{2}$ sont reportées sur la figure 4 .

L'EISF est d'autant plus proche de 1 que la particule est localisée. Par exemple pour la courbe supérieure avec $\tau_{1} / \tau_{2}=0.02$, la particule se trouve pratiquement localisée, présente pendant $98 \%$ du temps dans un site et $2 \%$ dans l'autre. Les sites sont indifférentiés et les mêmes courbes seraient obtenues par échange de $\tau_{1}$ et $\tau_{2}$, la délocalisation la plus forte étant obtenue pour $\tau_{l}=\tau_{2}$. 


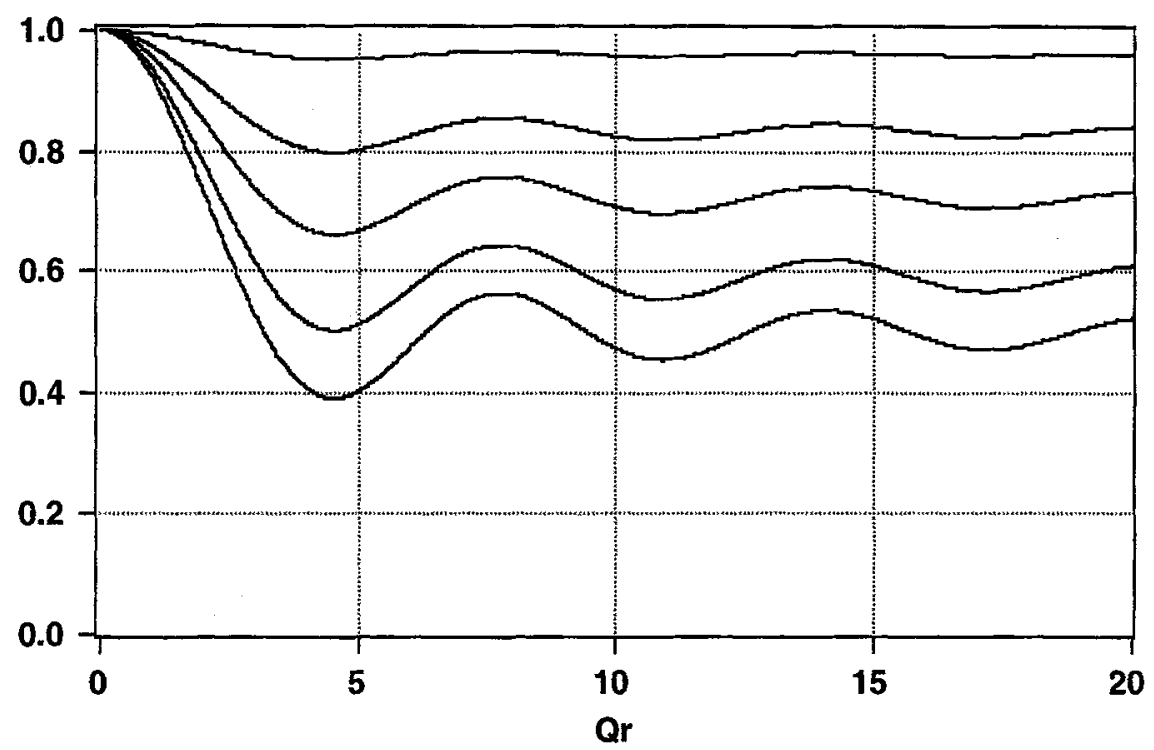

Figure 4 EISF de la réorientation sur deux sites non-équivalents pour différentes valeurs de $\tau_{1} / \tau_{2}$. De bas en haut, les courbes correspondent à des valeurs de $\tau_{\mathfrak{l}} / \tau_{2}$ égales à $1,0.4,0.2,0.1$ et 0.02 .

\subsection{Sauts sur trois sites équivalents}

Comme dans le premier cas, la dynamique est caractérisée par une seule constante de temps $\tau$, mais il est nécessaire d'élargir la définition du temps de résidence lorsqu'il y a plus de deux sites disponibles ; on définit de façon générale $\tau_{i j}^{-1}$ comme la fréquence moyenne de saut d'une particule du site $i$ vers le site $j$; dans le cas présent $\tau^{-1}=\tau_{12}^{-1}=\tau_{13}^{-1}$; la fréquence de sauts de 1 vers $2+3$ est $\tau_{r}^{-1}=\tau_{12}^{-1}+\tau_{13}^{-1}=2 / \tau$ et le temps de résidence moyen $\tau_{r}$ sur le site 1 est égal à $\tau / 2$.

Les équations de vitesse s'écrivent

$$
\begin{aligned}
& \frac{d}{d t} p_{1}(t)=\frac{1}{\tau}\left[-2 p_{1}(t)+p_{2}(t)+p_{3}(t)\right] \\
& \frac{d}{d t} p_{2}(t)=\frac{1}{\tau}\left[p_{1}(t)-2 p_{2}(t)+p_{3}(t)\right] \\
& \frac{d}{d t} p_{3}(t)=\frac{1}{\tau}\left[p_{1}(t)+p_{2}(t)-2 p_{3}(t)\right]
\end{aligned}
$$

A l'équilibre thermodynamique :

$$
\left\langle p_{1}\right\rangle=\left\langle p_{2}\right\rangle=\left\langle p_{3}\right\rangle=\frac{1}{3}
$$


Pour une poudre

$$
\begin{aligned}
& I(Q, t)=A_{0}(Q)+A_{1}(Q) \exp \left(-t / \tau_{c}\right) \\
& S(Q, \omega)=A_{0}(Q)+A_{1}(Q) \frac{1}{\pi} \frac{\tau_{c}}{1+\omega^{2} \tau_{c}^{2}}
\end{aligned}
$$

avec $\tau_{c}=\tau / 3$ le temps caractéristique et :

$$
\begin{aligned}
& A_{0}=\frac{1}{3}\left[1+2 j_{0}(Q d)\right] \\
& A_{l}(Q)=\frac{2}{3}\left[1-j_{0}(Q d)\right]
\end{aligned}
$$

Si $r$ est rayon du cercle dans lequel est inscrit le triangle équilatéral formé par les trois sites, on peut écrire $d=\left|\mathbf{r}_{2}-\mathbf{r}_{1}\right|=r \sqrt{3}$.

Dans les trois exemples considérés, la dynamique est caractérisée par un seul temps caractéristique $\tau_{c}$ et une seule distance de saut. La composante dynamique du facteur de structure incohérent se réduit à une seule lorentzienne de largeur à mi-hauteur $\tau_{c}$ et l'EISF $A_{o}(Q)$ est caractérisée par une seule fonction de Bessel sphérique $j_{0}(Q d)$ pour une poudre. La famille des cas très simples est complète si l'on ajoute la réorientation sur les quatre sommets d'un tétraèdre régulier pour lequel $\tau_{c}=\tau / 4$,

$$
A_{0}(Q)=\frac{1}{4}\left[1+3 j_{0}(Q d)\right] \text { et } A_{1}(Q)=\frac{3}{4}\left[1-j_{0}(Q d)\right]
$$

\section{GENERALISATION DE LA METHODE DE CALCUL}

A ce point il convient de résumer et de généraliser la méthode de traitement utilisée pour les cas simples. La fonction de diffusion intermédiaire est la moyenne du produit de la fonction de diffusion incohérente au temps $t$, $e^{i Q . r(t)}$, et la même fonction $e^{i Q . r(0)}$ à $t=0$. Dans les exemples étudiés, la résolution des équations différentielles a permis de séparer les composantes spatiales des composantes dynamiques, ainsi pour $n$ sites accessibles, la fonction est la somme de $n^{2}$ termes

$$
I(\mathbf{Q}, t)=\sum_{i=1}^{n}\left[\left\langle p_{i}\right\rangle \sum_{j=1}^{n} p_{i j}(t) e^{i \mathbf{Q} \cdot\left(r_{i}-\mathbf{r}_{i}\right)}\right]
$$

Chaque terme est le produit

- de la probabilité moyenne $\left\langle p_{i}\right\rangle$ d'occupation du site i (équilibre thermodynamique),

- de la probabilité dynamique de saut du site i vers le site $\mathbf{j}$, probabilité qui ne dépend pas de la position des sites.

- de la corrélation spatiale indépendante du temps. 
La corrélation spatiale sera toujours de la forme $\cos Q \cdot\left(r_{j}-r_{i}\right)$, ou $j_{0}(Q d)$ pour une poudre, avec $d=\left|r_{j}-r_{i}\right|$. Dans la suite, seuls les résultats pour les poudres seront explicités en détail, mais le retour au monocristal ne pose en principe pas de problème.

Le facteur dynamique $p_{i j}(t)$ est de la forme

$$
p_{i j}(t)=A_{i j}+\sum_{\alpha=0}^{n-1} B_{i j} e^{-t / \tau_{\alpha}}
$$

où les $\tau_{\alpha}$ sont des temps de corrélation caractéristiques obtenus à la résolution des $n$ équations différentielles .

Il y a formellement $n$ solutions aux équations différentielles, l'une d'elles sera toujours nulle (son facteur de forme est l'EISF) et les autres peuvent être dégénérées comme dans les cas simples étudiés jusqu'alors.

Pour les exemples plus complexes caractérisés par plusieurs fréquences ou/et plusieurs distances, la résolution « manuelle » devient rapidement fastidieuse et il convient d'utiliser les outils mathématiques appropriés.

La détermination des temps caractéristiques $\tau_{\alpha}$ revient à calculer les valeurs propres de la matrice $\mathrm{M}$ formée par les $n$ équations de vitesse, c'est-à-dire les racines de l'équation caractéristique $\lambda$ définie par la relation $\lambda I-M=0$.

Chaque solution est de la forme $p(t)=A+B e^{\lambda_{\alpha} t}$ avec $\lambda_{\alpha}=-1 / \tau_{\alpha}$.

De même l'EISF et les facteurs de forme des lorentziennes rendant compte de la dynamique sont obtenus en résolvant l'équation caractéristique des distances de sauts.

De façon générale la fonction intermédiaire s'écrira :

$$
I(Q, t)=\sum_{\alpha=0}^{n-1} A_{\alpha}(Q) e^{-t / \tau_{\alpha}}
$$

avec l'EISF égal à $A_{0}(Q)$ correspondant à $1 / \tau_{0}=0$

Sur la figure 5 est reportée la résolution de la réorientation sur les sommets d'un tétraèdre évoquée en $\mathrm{A} 3$ (équation 24) ; A4a est la matrice des équations de vitesse et $\mathrm{A} 4 \mathrm{aR}$ la matrice des facteurs de forme avec $A=j_{0}(Q d)$; les solutions doivent être multipliées par la probabilité thermodynamique d'occupation d'un site (1/4); on trouve une seule solution des équations dynamique trois fois dégénérée. L'EISF $A_{o}(Q)$ correspond à la solution $1 / \tau_{c}=0$ et $A_{l}(Q)$ est la somme des trois solutions correspondant au même temps caractéristique $4 / \tau$. On retrouve immédiatement les résultats des équations 24 . 


\section{COMBINAISON DE PLUSIEURS TYPES DE MOUVEMENT}

Dans ce paragraphe sont développés quelques exemples de réorientation sur des sites équidistants sur un cercle correspondant à des réorientations par sauts de $2 \pi / n$. On peut supposer que seuls les sauts sur sites voisins ont un sens physique (le saut sur un second voisin passant obligatoirement par le site proche voisin), mais il est formellement possible de résoudre le problème complet.

\subsection{Réorientation sur quatre sites équidistants sur un cercle}

En toute généralité, la dynamique est caractérisée par deux types de mouvements, sauts sur les sites voisins ou saut sur le site opposé. Ces sauts correspondent à des sauts sur sites voisins et site opposé ou à des rotations respectives de $\pi / 4,-\pi / 4$ et $\pi$ autour de l'axe de symétrie. Soit $1 / \tau_{4}$ et $1 / \tau_{2}$ les fréquences de saut correspondant à ces mouvements. On trouve quatre solutions $\lambda_{0}=0, \lambda_{1}=-\frac{4}{\tau_{4}}, \lambda_{2}=\lambda_{3}=-\frac{2}{\tau_{4}}-\frac{2}{\tau_{2}}$.

Pour une poudre, L'EISF égal à

$$
A_{0}(Q)=\frac{1}{4}\left[1+2 j_{0}(Q a)+j_{0}(Q a \sqrt{2})\right]
$$

et la composante quasiélastique est formée de deux lorentziennes de largeur $-1 / \lambda_{1}$ et $-1 / \lambda_{2}$ dont les facteurs de forme respectifs sont

$$
\begin{array}{ll}
A_{1}(Q) & =\frac{1}{2}\left[1-j_{0}(Q a \sqrt{2})\right] \\
\text { et } \quad A_{2}(Q) & =\frac{1}{4}\left[1-2 j_{0}(Q a)+j_{0}(Q a \sqrt{2})\right]
\end{array}
$$

Les exemples simples choisis traitent de façon assez formelle le saut de particules sur des sites. En pratique, ces particules font souvent partie d'une molécule et les réorientations se produisent par rotation autour d'un axe ; le saut sur quatre sites devient une rotation sur un cercle avec quatre positions équivalentes et les sauts entre sites opposés sont souvent peu vraisemblables. On écrit les équations de vitesse avec $2 / \tau_{2}=0$; les solutions sont du même type que dans le cas général en supprimant $-2 / \tau_{2}$ de la solution $\lambda_{2}$, ainsi $\lambda_{2}=\lambda_{3}=-2 / \tau_{4}$. L'EISF et les facteurs de forme ne sont pas modifiés mais on peut remplacer $a$ par $r \sqrt{2}, r$ étant le rayon du cercle.

\subsection{Réorientation sur $n$ sites équidistants sur un cercle}

La généralisation à la réorientation sur $n$ sites équivalents sur un cercle en n'acceptant les échanges qu'entre proches voisins donne

$$
\frac{1}{\tau_{\alpha}}=\frac{4}{\tau} \sin ^{2}\left(\frac{\alpha \pi}{N}\right)
$$

Note. Il est important de remarquer dans ce dernier cas que la dynamique du système est contrôlée par une seule fréquence de saut $1 / \tau_{4}$ correspondant à un seul type de mouvement entre sites voisins mais qque le spectre quasiélastique présente deux fréquences caractéristiques $4 / \tau_{4}$ et $2 / \tau_{4}$. 


\section{quatre sites équivalents (tétraèdre)}

$>$ with(linag) ;

$>A 4 a:=$ matrix $([[-3 / \operatorname{tau}, 1 /$ tau,1/tau,1/tau],[1/tau,-3/tau, $1 /$ tau, $1 /$ tau $],[1 /$ tau, $1 /$ tau,, $3 /$ tau, $1 /$ tau $]$, $[1 /$ tau, $1 /$ tau, $1 /$ tau,,$-3 /$ tau $]])$;

$$
A 4 a=\left[\begin{array}{cccc}
-3 \frac{1}{\tau} & \frac{1}{\tau} & \frac{1}{\tau} & \frac{1}{\tau} \\
\frac{1}{\tau} & -3 \frac{1}{\tau} & \frac{1}{\tau} & \frac{1}{\tau} \\
\frac{1}{\tau} & \frac{1}{\tau} & -3 \frac{1}{\tau} & \frac{1}{\tau} \\
\frac{1}{\tau} & \frac{1}{\tau} & \frac{1}{\tau} & -3 \frac{1}{\tau}
\end{array}\right]
$$

$>$ eigenvals(A4a);

$$
0 .-4 \frac{1}{\tau}-4 \frac{1}{\tau}-4 \frac{1}{\tau}
$$

A4aR:=matrix $([[1, A, A, A],[A, 1, A, A],[A, A, 1, A],[A, A, A, 1]])$

$$
A \operatorname{AaR}:=\left[\begin{array}{llll}
1 & A & A & A \\
A & 1 & A & A \\
A & A & 1 & A \\
A & A & A & 1
\end{array}\right]
$$

$>$ eigenvals(A4aR);

$$
3 A+1-A+1-A+1-A+1
$$

Figure 5. Résolution de la réorientation sur les sommets d'un tétraèdre en utilisant la méthode de résolution matricielle et le logiciel Maple V. A4a est la matrice dynamique et A4aR la matrice des facteurs de forme avec $A=j_{0}(Q d)$. La fonction eigenvals $(M)$ donne les valeurs propres de $M$. 
Le spectre quasi élastique est formé de $\mathrm{n} / 2$ lorentziennes si le nombre de sites est pair et (n-1)/2 s'il est impair. Dans la littérature, il existe une certaine ambiguiité, déjà évoquée plus haut, sur la définition de $\tau$. Nous gardons tout au long de l'exposé la même définition, à savoir $1 / \tau_{\mathrm{ij}}$ est la fréquence des sauts depuis le site $i$ vers le site $j$, avec $\tau=\tau_{\mathrm{ij}}=\tau_{\mathrm{ji}}$ quand il n'y a qu'un seul temps mis en jeu. Le temps de résidence moyen sur un site est ainsi déterminé de manière générale et univoque par la relation

$$
\frac{1}{\tau_{i}}=\sum_{j \neq i=1}^{n-1} \frac{1}{\tau_{i j}}
$$

Les facteurs de structure correspondants s'écrivent :

$$
A_{l}(Q)=\frac{1}{n} \sum_{\alpha=1}^{n} j_{0}\left(Q d_{\alpha}\right) \cos \left(\frac{2 \alpha l \pi}{n}\right)
$$

avec la distance de saut $d_{\alpha}$ exprimée en fonction du rayon $r$ du cercle par $d_{\alpha}=2 r \sin \left(\frac{\alpha \pi}{N}\right)$.

Par exemple, pour une réorientation avec sauts entre plus proches voisins sur 6 sites équivalents, il existe trois solutions non nulles aux équations de vitesses $\lambda_{1}=-1 / \tau, \lambda_{2}=-3 / \tau$ et $\lambda_{3}=-4 / \tau$.

Les facteurs de structure correspondants qui son reportés sur la figure 6 sont les suivants

$$
\begin{aligned}
& A_{0}(Q)=\frac{1}{6}[1+2 A+2 B+C] \\
& A_{1}(Q)=\frac{1}{3}[1+A-B-C] \\
& A_{2}(Q)=\frac{1}{3}[1-A-B+C] \\
& A_{3}(Q)=\frac{1}{6}[1-2 A+2 B-C]
\end{aligned}
$$

avec

$$
A=j_{0}(Q r), B=j_{0}(Q r \sqrt{3}), C=j_{0}(2 Q r)
$$

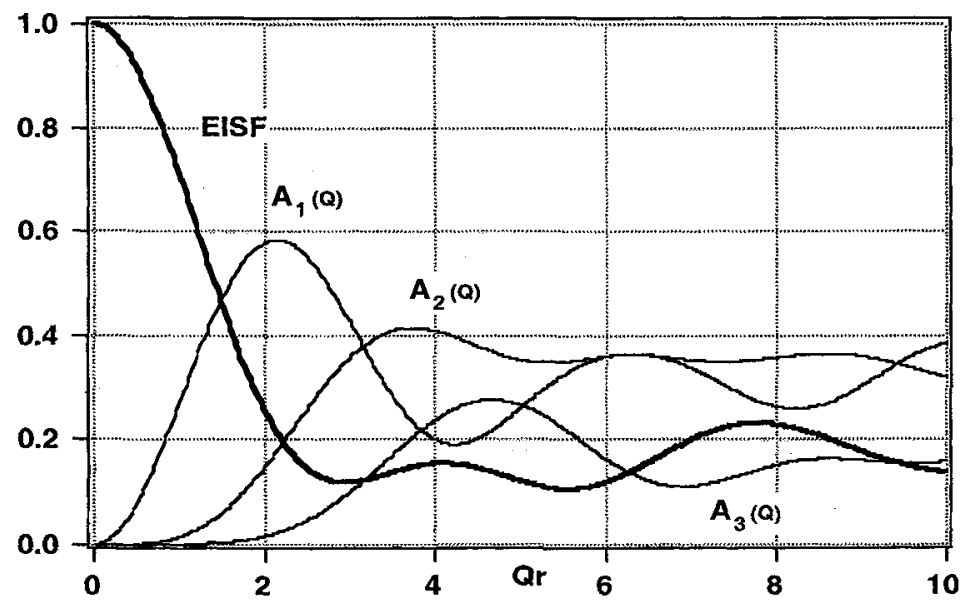

Figure 6. EISF et les trois facteurs de forme correspondant à la réorientation sur six sites équivalents disposés sur un cercle. 


\section{UTILISATION DES GROUPES DE SYMETRIE}

La méthode de résolution matricielle utilisée dans les paragraphes précédents a permis de rendre compte des réorientations de groupements dans un plan perpendiculaire à un axe de rotation; elle peut également être appliquée à des réorientations hors du plan, mais reste insuffisante, ou deviendra trop complexe, pour étudier toutes les réorientations possibles d'une molécule dans son site cristallin; en effet la méthode traite en fait de réorientations par sauts et ne fait pas apparaître explicitement les rotations . La méthode générale utilisera la théorie des groupes qui tient compte de tous les mouvements possibbles autour des axes de symétrie. Par exemple la réorientation d'une molécule autour d'un axe C3 d'un système cubique peut mettre en jeu plusieurs distances de saut pour un même temps caractéristique; dans la rotation de $2 \pi / 3$, les deux atomes situées au sommet du cube et sur l'axe de rotation ne bougent pas, les atomes situés sur les autres sommets parcourent une distance $a \sqrt{2}$, les atomes situés au centre des faces $a / \sqrt{2}$; de plus un atome peut utiliser plusieurs chemins pour passer d'un site à un autre.

L'utilisation de la théorie des groupes exige que la probabilité d'occupation de tous les sites qui peuvent être visités par une particule soit identique, c'est-à-dire $\left\langle\mathrm{p}_{\mathrm{i}}\right\rangle=1 / \mathrm{g}$, g étant l'ordre du groupe, ce qui implique en particulier la symétrie $\tau_{\mathrm{ij}}=\tau_{\mathrm{ji}}$.

L'équation dynamique peut être réécrite de façon générale (équation de Fokker-Planck)

$$
\frac{d}{d t} P(R, t)=\sum_{S} f\left(R S^{-1}\right) P(S, t)-f(S) P(R, t)
$$

$P(R, t)$ est la probabilité que la particule ait été amenée, par la rotation $R$, de la position qu'elle avait à à $t=0$ vers sa position actuelle à $t$. La relation prend en compte tous les chemins possibles allant vers l'état final caractérisé par une distance de saut et une fréquence $f\left(R S^{-1}\right)$. La correspondance avec les équations de vitesse écrites précédemment est immédiate, la fréquence $f$ étant homogène à $1 / \tau$. La démarche du calcul est semblable à la méthode utilisée plus haut, en travaillant sur les matrices des représentations irréductibles dont la dimension est égale au nombre de classes du groupe, inférieur à l'ordre du groupe.

Un groupe est représenté par un certain nombre de classes de symétrie (identité, rotation autour d'axes $\mathrm{C}_{4}, \mathrm{C}_{3}, \mathrm{C}_{2} \ldots$ ) et par un nombre équivalent de représentations irréductibles. A chaque classe $\mu$ correspondent une ou plusieurs distances de saut, à chaque représentation irréductible $\Gamma_{\alpha}$ correspond une fréquence de saut $1 / \tau_{\alpha}$.

Les temps caractéristiques sont déterminés en trouvant les racines de l'équation caractéristique pour chaque représentation irréductible ; pour cela il est suffisant de connaître le caractère $\chi_{\alpha}$ qui est la trace de la représentation irréductible $\Gamma_{\alpha}$. Ainsi

$$
\frac{1}{\tau_{\alpha}}=\sum_{\mu} \frac{1}{\tau_{\mu}}\left(1-\frac{\chi_{\alpha}^{\mu}}{\chi_{\alpha}^{E}}\right)
$$

chaque classe de rotation $\mu$ est caractérisé par la fréquence $1 / \tau_{\mu}$. 
La fonction de diffusion intermédiaire prend la forme générale

avec

$$
I(Q, t)=\sum_{\alpha} A_{\alpha}(Q) e^{-t / \tau_{\alpha}}
$$

et pour une poudre

$$
\begin{aligned}
& A_{\alpha}(Q)=\frac{\chi_{\alpha}^{E}}{g} \sum_{\mu, R_{\mu}} \chi_{\alpha}^{\mu} e^{i Q \cdot\left(r-R_{\mu} r\right)} \\
& A_{\alpha}(Q)=\frac{\chi_{\alpha}^{E}}{g} \sum_{\mu} \chi_{\alpha}^{\mu} j_{0}\left(Q d_{\mu}\right)
\end{aligned}
$$

\subsection{Application à la réorientation sur 4 sites équivalents sur un cercle (Group $\left.\mathrm{C}_{4}\right)$}

Le groupe comprend quatre classe $E, C_{4}, C_{2}, C_{4}^{3}$ correspondant à la matrice de rotation suivante

\begin{tabular}{c|cccc} 
& $E$ & $C_{4}$ & $C_{2}$ & $C_{4}^{3}$ \\
\hline$E$ & $E$ & $C_{4}$ & $C_{2}$ & $C_{4}^{3}$ \\
$C_{4}$ & $C_{4}$ & $C_{2}$ & $C_{4}^{3}$ & $E$ \\
$C_{2}$ & $C_{2}$ & $C_{4}^{3}$ & $E$ & $C_{4}$ \\
$C_{4}^{3}$ & $C_{4}^{3}$ & $E$ & $C_{4}$ & $C_{2}$
\end{tabular}

On peut consulter les tables des caractères dans beaucoup d'ouvrages de mathématique, de cristallographie ou de spectroscopie; dans le cas des groupes cycliques $C_{n}$ (définis par $C^{n}=E$ ) les caractères s'écrivent

$$
\chi_{\alpha}^{\mu}=e^{i \frac{2 \pi \alpha \mu}{n}}
$$

La table des caractères du groupe $\mathrm{C}_{4}$ est reportée sur le tableau 1. Dans la dernière colonne sont reportées les valeurs de chaque représentation irréductible calculés à partir de 34; dans la ligne du bas est reportée la seule distance du saut $d_{\mu}$ de chaque rotation. Bien que n'appartenant pas à la même classe, les rotations $\mathrm{C}_{4}$ et $\mathrm{C}_{43}=-\mathrm{C}_{4}$ ont le même effet, donc la même distance de saut ; la conséquence est qu'à $\Gamma_{1}$ et $\Gamma_{3}$ correspond le même temps caractéristique $\tau_{\alpha}$.

\begin{tabular}{c|cccc|c} 
& $E$ & $C_{4}$ & $C_{2}$ & $C_{4}^{3}$ & $\frac{1}{\tau_{\alpha}}$ \\
\hline$\Gamma_{0}$ & 1 & 1 & 1 & 1 & 0 \\
$\Gamma_{1}$ & 1 & $i$ & -1 & $-i$ & $\frac{2}{\tau_{4}}+\frac{2}{\tau_{2}}$ \\
$\Gamma_{2}$ & 1 & -1 & 1 & -1 & $\frac{4}{\tau_{4}}$ \\
$\Gamma 3$ & 1 & $-i$ & -1 & $i$ & $\frac{2}{\tau_{4}}+\frac{2}{\tau_{2}}$ \\
\hline$d_{j}$ & 0 & $r \sqrt{2}$ & $2 r$ & $r \sqrt{2}$ &
\end{tabular}

Tableau 1- table des caractères du groupe $\mathrm{C}_{4}$ 
En utilisant la relation 37, on retrouve immédiatement les facteurs de structure $A_{\alpha}(Q)$ calculés au paragraphe B1.

\subsection{Exemples d'application au groupe cubique}

La table des caractère du groupe $O_{h}$ s'écrit

\begin{tabular}{c|ccccc} 
& $E$ & $8 C_{3}$ & $3 C_{4}^{2}$ & $6 C_{4}$ & $6 C_{2}$ \\
\hline$A_{1}$ & 1 & 1 & 1 & 1 & 1 \\
$A_{2}$ & 1 & 1 & 1 & -1 & -1 \\
$E$ & 2 & -1 & 2 & 0 & 0 \\
$T_{1}$ & 3 & 0 & -1 & 1 & -1 \\
$T_{2}$ & 3 & 0 & -1 & -1 & 1
\end{tabular}

Les fréquences caractéristiques du système sont calculées à partir de l'équation 34

$$
\begin{aligned}
& \frac{1}{\tau_{A_{1}}}=0 \\
& \frac{1}{\tau_{A_{2}}}=\frac{2}{\tau_{C_{4}}}+\frac{2}{\tau_{C_{2}^{\prime}}} \\
& \frac{1}{\tau_{E}}=\frac{3}{2 \tau_{C_{3}}}+\frac{1}{\tau_{C_{4}}}+\frac{1}{\tau_{C_{2}^{\prime}}} \\
& \frac{1}{\tau_{T_{1}}}=\frac{1}{\tau_{C_{3}}}+\frac{2}{3 \tau_{C_{4}}}+\frac{4}{3 \tau_{C_{4}^{2}}}+\frac{4}{3 \tau_{C_{2}^{\prime}}} \\
& \frac{1}{\tau_{T_{2}}}=\frac{1}{\tau_{C_{3}}}+\frac{4}{3 \tau_{C_{4}}}+\frac{4}{3 \tau_{C_{4}^{2}}}+\frac{2}{3 \tau_{C_{2}^{\prime}}}
\end{aligned}
$$

Les facteurs de forme correspondants peuvent se mettre sous la forme compacte suivante

$$
\begin{aligned}
& A_{1}(Q)=\frac{1}{24}[1+2 A+B+C+2 D] \\
& A_{2}(Q)=\frac{1}{24}[1+2 A+B-C-2 D] \\
& E(Q)=\frac{4}{24}[1-A+B] \\
& T_{1}(Q)=\frac{3}{24}[3-B+C-2 D] \\
& T_{2}(Q)=\frac{3}{24}[3-B-C+2 D]
\end{aligned}
$$

où les coefficients $\mathrm{A}$ à $\mathrm{D}$ donnent les contributions de chaque type de rotation $\left(C_{3}, C_{4}^{2}, \dot{C}_{2}\right.$ et $C_{4}$ respectivement). Dans cette représentation chaque fonction ( $A$ à $D$ ) peut être la somme de plusieurs 
distances de réorientation. Les relations pour une position générale du groupe cubique est donnée dans Marc Bée (page 223)

Considérons le cas d'atomes situés sur les sommets d'un cube de côté $a$ :lesfacteurs de forme sont les suivants :

$$
\begin{array}{ll}
2 A=2+6 j_{0}(Q a \sqrt{2}) & \text { rotation } C_{3}, \quad 8 \text { distances mais } 2 \text { atomes restent fixes } \\
B=3 j_{0}(Q a \sqrt{2}) & \text { rotation } C_{4}^{2}, \quad 3 \text { distances équivalentes } \\
C=3 j_{0}(Q a)+3 j_{0}(Q a \sqrt{3}) & \text { rotation } C_{2}, \quad 6 \text { distances } \\
2 D=6 j_{0}(Q a) & \text { rotation } C_{4}, \quad 6 \text { distances équivalentes }
\end{array}
$$

ce qui donne

$$
\begin{aligned}
& A_{1}(Q)=\frac{1}{8}\left[1+3 j_{0}(Q a)+3 j_{0}(Q a \sqrt{2})+j_{0}(Q a \sqrt{3})\right] \\
& A_{2}(Q)=\frac{1}{8}\left[1-3 j_{0}(Q a)+3 j_{0}(Q a \sqrt{2})-j_{0}(Q a \sqrt{3})\right] \\
& E(Q)=0 \\
& T_{1}(Q)=\frac{3}{8}\left[1+j_{0}(Q a)-j_{0}(Q a \sqrt{2})-j_{0}(Q a \sqrt{3})\right] \\
& T_{2}(Q)=\frac{3}{8}\left[1-j_{0}(Q a)-j_{0}(Q a \sqrt{2})+j_{0}(Q a \sqrt{3})\right]
\end{aligned}
$$

La résolution matricielle pour la réorientation entre sites voisins est donnée en annexe 1

\section{2a Application à la réorientation sur les sommets d'un tétraèdre}

La réorientation sur les sommets d'un tétraèdre interdit les rotations $C_{4}$ et $C_{2}$; ainsi $\frac{1}{\tau_{A_{2}}}=0$ et $\frac{1}{\tau_{T_{1}}}=\frac{1}{\tau_{T_{2}}}=\frac{2}{\tau_{C_{3}}}+\frac{2}{\tau_{C_{4}^{2}}}$.

L'EISF sera ainsi la somme de $A_{1}(Q)+A_{2}(Q)$ soit

$$
A_{0}(Q)=\frac{1}{4}\left[1+3 j_{0}(Q a \sqrt{2})\right]
$$

Comme les temps caractéristiques des modes de $T_{1}$ et $T_{2}$ sont identiques, la diffusion quasiélastique est formée d'une seule lorentzienne dont le facteur de forme est égal à la somme $T_{1}(Q)+T_{2}(Q)$

$$
A_{1}(Q)=\frac{3}{4}\left[1-j_{0}(Q a \sqrt{2})\right]
$$

note_ On retrouve le résultat obtenu au paragraphe A3. La fréquence caractéristique était alors la fréquence de saut d'un site vers chacun des trois autres sites équivalents. En utilisant la théorie des groupes, cette même fréquence apparaît comme la somme de deux types de rotation sans possibilité de déterminer la contribution de chacune. Cette discussion est un peu formelle; en pratique, si la rotation $\mathrm{C}_{4}$ est interdite, il est fort probable que la rotation $C_{4}^{2}$ le soit également. 


\section{2b Réorientation sur les sommets d'un octaèdre (proches voisins)}

Les atomes sont positionnés sur les centres des faces d'un cube ; la réorientation sur les seuls proches voisins n' autorise que les rotations $C_{3}$ et $C_{4}$. A partir de $40 \mathrm{~b}$, on trouve :

$$
\begin{array}{ll}
2 A=8 j_{0}(Q a \sqrt{2} / 2) & \text { rotation } C_{3}, 8 \text { distances équivalentes } \\
B=1+2 j_{0}(Q a) & \text { rotation } C_{4}^{2}, 3 \text { distances mais } 1 \text { atome reste fixé } \\
C=4 j_{0}(Q a \sqrt{2} / 2)+2 j_{0}(Q a) & \text { rotation } C_{2}^{\prime}, 2 \text { types de distance } \\
2 D=2+4 j_{0}(Q a \sqrt{2} / 2) & \text { rotation } C_{4}, 2 \text { atomes restent inchangés }
\end{array}
$$

ce qui donne

$$
\begin{aligned}
& A_{1}(Q)=\frac{1}{6}\left[1+4 j_{0}(Q a \sqrt{2})+j_{0}(Q a)\right] \\
& A_{2}(Q)=0 \\
& E(Q)=\frac{1}{3}\left[1-2 j_{0}(Q a \sqrt{2} / 2)+j_{0}(Q a)\right] \\
& T_{1}(Q)=0 \\
& T_{2}(Q)=\frac{1}{2}\left[1-j_{0}(Q a)\right]
\end{aligned}
$$

Le spectre quasiélastique est composé de deux lorentziennes dont les fréquences caractéristiques sont égales à $\frac{1}{\tau_{1}}=\frac{3}{2 \tau_{C_{3}}}+\frac{1}{\tau_{C_{4}}} \quad$ et $\frac{1}{\tau_{2}}=\frac{1}{\tau_{C_{3}}}+\frac{4}{3 \tau_{C_{4}}}$ correspondant respectivement aux facteurs de forme $E(Q)$ et $T_{2}(Q)$.

\footnotetext{
Note. La réorientation par sauts sur premiers voisins ne met en jeu qu'un seul temps de relaxation $\tau$. La résolution matricielle de ce système (Annexe 2) donne deux fréquences caractéristiques égales à $6 / \tau$ et $4 / \tau$ qui peuvent être comparées aux valeurs calculées ci dessus. Comme dans le cas du tétraèdre, si la réorientation se fait par sauts, la dynamique est caractérisée par un seul temps $\tau$ donnant lieu à deux lorentziennes; si la réorientation se fait par rotation, le mouvement met en jeu deux fréquences correspondant aux rotations $C_{3}$ et $C_{4}$, toutes deux physiquement significatives cette fois. Le spectre quasiélastique ne permet pas de séparer les deux contributions. L'EISF et les deux facteurs de forme $A_{l}(Q)$ et $A_{2}(Q)$ sont reportés sur la figure 7.
} 


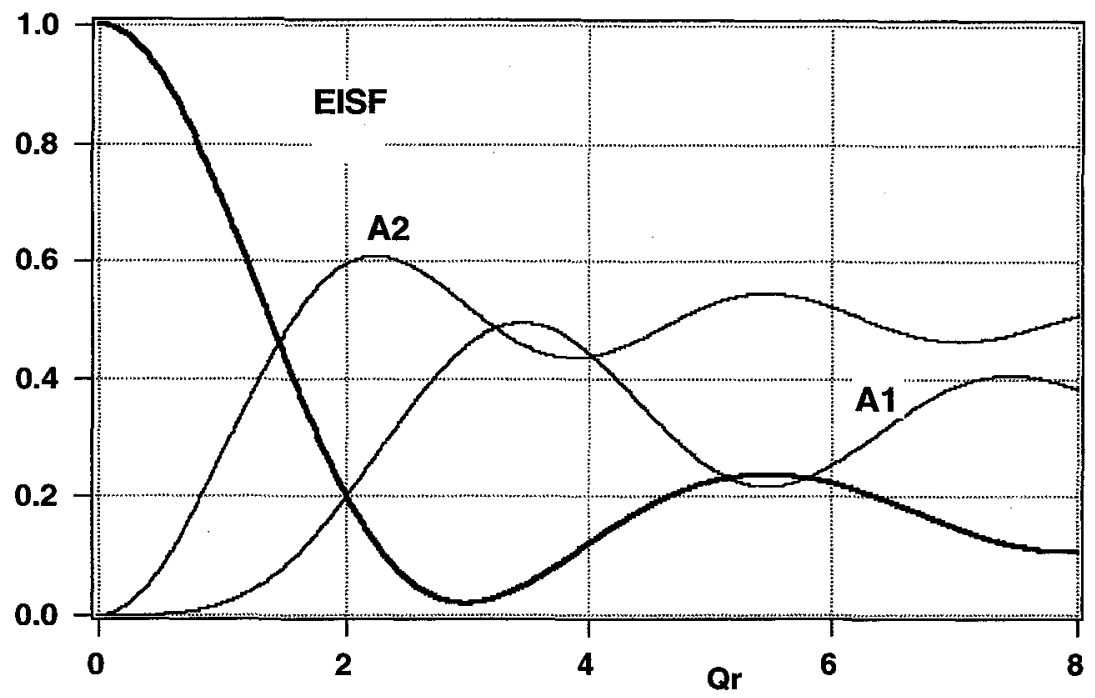

Figure 7. EISF et facteur de forme des deux courbes lorentziennes pour la réorientation sur les sommets d'un octaèdre régulier

\section{MOUVEMENTS DE REORIENTATION CONTINUS.}

Lorsque les potentiels locaux s'opposant à la rotation sont nuls ou très faibles, on peut observer des mouvements de rotations continus autour d'un axe ou par rapport au centre de symétrie.

Le problème revient à résoudre la loi de diffusion de Fick rotationnelle. Nous considérerons le cas d'une rotation isotrope sur un cercle autour d'un axe de symétrie, la réorientation isotrope à la surface d'une sphère, et enfin la diffusion isotrope dans un volume à l'intérieur d'une sphère.

\subsection{Diffusion uniaxiale isotrope.}

L'équation de Fick s'écrit

$$
\frac{\partial P}{\partial t}=D_{r} \frac{\partial^{2} P}{\partial \varphi^{2}}
$$

dont la solution est

$$
P(\varphi, t)=\frac{1}{2 \pi} \sum_{n=-\infty}^{n=\infty} e^{i n\left(\varphi-\varphi_{0}\right) t} e^{-n^{2} D_{r} t}
$$

où $\varphi_{0}$ est la position angulaire à $t=0$ et $D_{r}$ est le coefficient de diffusion rotationnel. 
La fonction de diffusion intermédiaire est donnée par

$$
I_{i n c}(\mathbf{Q}, t)=\int_{0}^{2 \pi} e^{i \mathrm{Q} . \mathrm{r}(t)} e^{-i \mathrm{Q} \cdot \mathbf{r}(0)} P(\varphi, t)\langle P\rangle d \varphi d \varphi_{0}
$$

où $\langle P\rangle$ correspond à l'équilibre thermodynamique ; dans le cas de la diffusion isotrope sur un cercle,

$$
\langle P\rangle=1 / 2 \pi
$$

La position du vecteur $Q$ par rapport à la direction de l'axe directeur du cercle peut être définie par un angle polaire $\theta$ et un angle azimutal $\psi$. Si $r$ est le rayon du cercle, les produits scalaires entre Q et $\mathbf{r}$ deviennent

$$
\begin{aligned}
& \text { Q.r }(\varphi)=Q r \sin \theta \cos (\varphi-\psi) \\
& \text { Q.r }\left(\varphi_{0}\right)=Q r \sin \theta \cos \left(\varphi_{0}-\psi\right)
\end{aligned}
$$

En utilisant le développement

$$
e^{i x \cos \alpha}=\sum_{m=-\infty}^{+\infty} i^{m} e^{-i m \alpha} J_{m}(x)
$$

où $J_{m}$ est la fonction de Bessel cylindrique d'ordre $m$, la fonction de diffusion intermédiaire devient

$$
I_{i n c}(\mathbf{Q}, t)=\sum_{m=-\infty}^{\infty} J_{m}^{2}(Q r \sin \theta) e^{-D, m^{2} t}
$$

et le facteur de structure incohérent

$$
S(\mathbf{Q}, \omega)=J_{0}^{2}(Q r \sin \theta) \delta(\omega)+2 \sum_{m=1}^{\infty} J_{m}^{2}(Q r \sin \theta) \times \frac{1}{\pi} \frac{D_{r} m^{2}}{\left(D_{r} m^{2}\right)^{2}+\omega^{2}}
$$

Le spectre quasiélastique est la superposition d'un nombre infini de courbes lorentziennes; en pratique seul un petit nombre de ces courbes contribuent au facteur de structure dans la fenêtre de mesure ; en effet, la surface sous chaque courbe diminue comme $1 / \mathrm{m}^{2}$ et la largeur varie en $\mathrm{m}^{2}$, de plus pour les ordres supérieurs à sept, la valeur des fonctions de Bessel est pratiquement nulle lorsque l'argument ( $\operatorname{Qr} \sin \theta)$ est inférieur à 5 . (figure 3)

Il n'existe pas d'expression analytique pour la moyenne de poudre qui est obtenue numériquement en intégrant les facteurs de forme par rapport à $\theta$. 


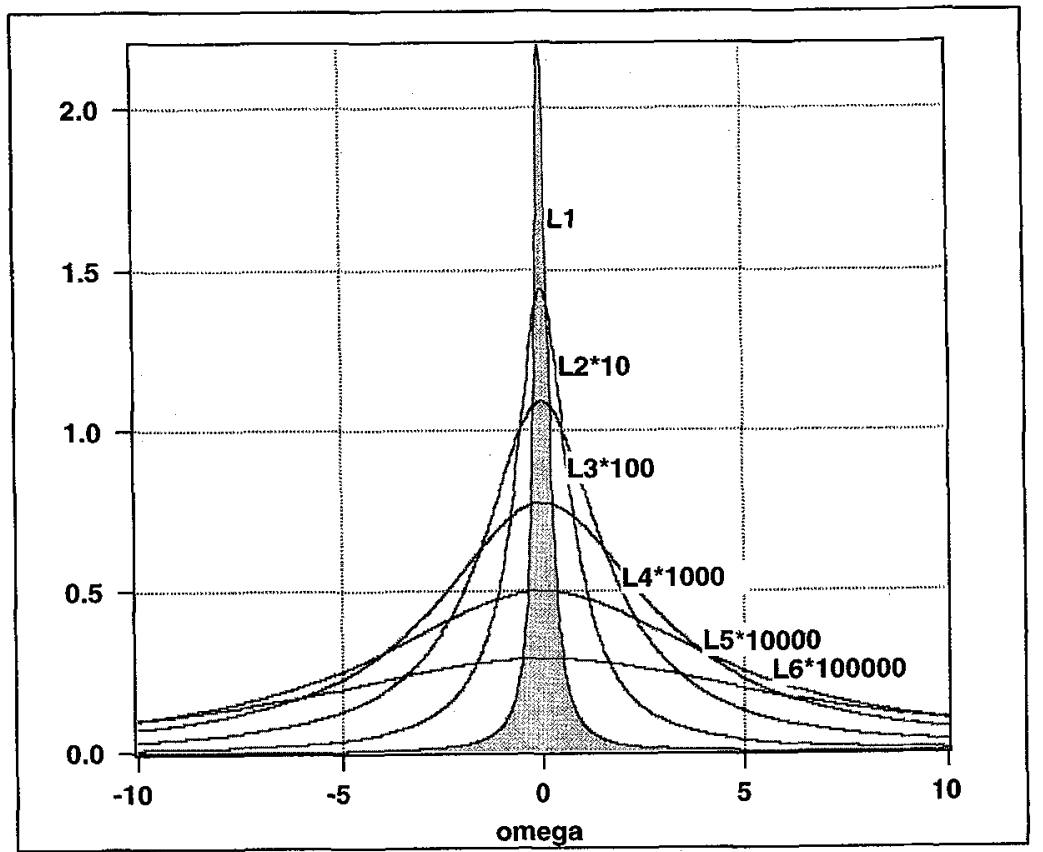

Figure 8 - rotation uniaxiale. Superposition des lorentziennes dans le massif quasiélatique ; l'argument de la fonction de Bessel $Q r \sin \theta$ est égal à 1 . L'amplitude des différentes courbes a été amplifié pour rendre toutes les courbes visibles

\subsection{Diffusion sur une sphère}

L'équation de Fick orientationnelle à trois dimensions s'écrit

$$
\frac{\partial}{\partial t} P(\Omega, t)=D_{r} \Delta_{\Omega} P(\Omega, t)
$$

où $D_{\mathrm{r}}$ est le coefficient de diffusion rotationnel

et $\Delta_{\Omega}$ est le Laplacien écrit en coordonnées sphériques (avec $r$ constant) ou en fonction des angles d 'Euler.

Dans le cas où la distribution sur la surface de la sphère est uniforme $(1 / 4 \pi)$, la loi de diffusion correspondante pour une poudre s'écrit :

$$
S(Q, \omega)=j_{0}^{2}(Q r) \delta(\omega)+\sum_{i=1}^{\infty} \frac{1}{\pi} \frac{l(l+1) D_{r}}{\left[l(l+1) D_{r}\right]^{2}+\omega^{2}}
$$

Comme dans le cas de la rotation sur un cercle, l'intensité quasiélastique est la somme d'un nombre infini de lorentzienne, mais seuls les tout premiers ordres ont une contribution significative. 


\subsection{Diffusion à l'intérieur d'une sphère}

La diffusion des molécules en milieu confiné est une partie importante de l'étude des mouvements localisés. Comme le produit des probabilités de $e^{i Q \cdot(t)} \mathbf{a} t=0$ et $t=\infty$ n'est pas nul, le spectre sera composé d'une partie élastique(EISF) et d'un massif quasiélastique. L'EISF décrit la taille et la forme de la cavité. Le massif quasiélastique est composé d'une série de lorentziennes dont la largeur grandit rapidement.

L'expression de l'EISF est simple

$$
A_{0}(Q)=\left(\frac{3 j_{1}(Q a)}{Q a}\right)^{2}
$$

avec $a$ le rayon de la sphère

et $\quad j_{1}(x)=\frac{\sin x}{x^{2}}-\frac{\cos x}{x}$ est la fonction de Bessel sphérique du premier ordre.

Cette relation présente un grand intérêt; en effet, si la forme de la cavité ne s'éloigne pas trop de la sphère, la relation 51 est le premier ordre d'une distribution plus complexe mais donne, de façon correcte, la taille moyenne de la cavité.

\section{CONCLUSION}

L'étude des mouvements localisés par diffusion de neutron est une discipline très riche. La mesure procure en effet des informations dynamiques précises sur les mouvements des atomes en réorientation dans les cristaux ; les facteurs de forme, dépendant de l'angle apportent des informations uniques sur la localisation des mouvements. Comme on l'a montré dans cette introduction, la modélisation mathématique du facteur de structure dynamique $S(Q, \omega)$ permet dans beaucoup de cas de proposer un petit nombre de paramètres dont la valeur pourra être directement déterminée à partir de la mesure par un programme de raffinement.

Trois directions peuvent être proposées pour développer un cours plus approfondi :

- l'aspect expérimental qui n'a pas été évoqué ici. Une expérience est réalisée dans une certaine fenêtre d'échange d'énergie et de moment de transfert. La résolution du spectromètre limite la détermination des mouvements lents; pour des fréquences au-dessous de la résolution, le spectromètre considère que les mouvements sont figés. A l'autre extrémité, les mouvements rapides échappent à la mesure, ce qui pose des problèmes de normalisation. En particulier l'extraction et la discussion sur l'EISF devront tenir compte de ces limitations expérimentales. La détermination et la modélisation précise de l'EISF est également tributaire de la limitation en transfert de moment, pour une poudre $Q_{\max }=4 \pi / \lambda$. La forme de l'EISF aux petits valeurs de $Q$ renseigne surtout sur la dimension globale du mouvement, par exemple le rayon du cercle ou de la sphère ; les détails sont apportés par les plus grandes valeurs de $Q$. Par exemple, pour $Q_{\max }=2$, il est difficile de distinguer la diffusion continue des mouvements de réorientations par sauts sur 4 sites sur un cercle de $1 \AA ̊$ de rayon. 
- les modèles hybrides. En plus de modèles réorientationnels plus riches que ceux qui ont été abordés ici, la résolution de modèles hybrides entre les sauts entre sites ponctuels et les modèles de diffusion continue est un domaine qui a été exploité avec succès dans de nombreux cas et qui reste en développement. Bien que très riches pour aider à la compréhension, les modèles analytiques feront souvent place aux simulations numériques qui apportent maintenant une aide inestimable au dépouillement des données.

- la comparaison avec les autres données structurales. Les données structurales obtenues par la détermination de l'EISF peuvent être complétées par les mesures de la diffusion diffuse obtenus sur un diffractomètre de poudre classique dont l'extension en $Q$ est plus grande.

\section{LECTURES}

Neutron Scattering Studies

A.J. Leadbetter et R.R. Lechner, dans « the plastically crystalline state » John Wiley \& sons, Chichester 1979

F. Guillaume

Thèse de Doctorat

Bordeaux, 1988

Quasielastic Neutron Scattering

M. Bée

Adam Hilger - Bristol 1988 


\section{Annexe 1}

\section{Huit sites sur un cube, sauts sur voisins ( $1 \tau$ )}

$>A 8:=$ matrix $([[-3 / \operatorname{tau}, 1 / \operatorname{tau}, 1 / \operatorname{tau}, 1 / \operatorname{tau}, 0,0,0,0],[1 / \operatorname{tau},-3 / \operatorname{tau}, 0,0,1 / \operatorname{tau}, 0,1 / \operatorname{tau}, 0],[1 / \operatorname{tau}, 0,-$ $\left.3 / \operatorname{tau}, 0,1 / \operatorname{tau}, 1 / \operatorname{tau}_{,}, 0,0\right],[1 / \tan , 0,0,-3 / \operatorname{tau}, 0,1 / \operatorname{tau}, 1 / \operatorname{tau}, 0],[0,1 / \operatorname{tau}, 1 / \operatorname{tau}, 0,-3 / \operatorname{tau}, 0,0,1 / \operatorname{tau}],[0,0,1 / \operatorname{tau}, 1 / \operatorname{tau}, 0,-$ $3 / \operatorname{tau}, 0,1 / \tan ],[0,1 / \operatorname{tau}, 0,1 / \operatorname{tau}, 0,0,-3 / \tan , 1 / \operatorname{tau}],[0,0,0,0,1 / \tan , 1 / \operatorname{tau}, 1 / \tan ,-3 / \operatorname{tau}]])$;

$$
A B=\left[\begin{array}{cccccccc}
-3 \frac{1}{\tau} & \frac{1}{\tau} & \frac{1}{\tau} & \frac{1}{\tau} & 0 & 0 & 0 & 0 \\
\frac{1}{\tau} & -3 \frac{1}{\tau} & 0 & 0 & \frac{1}{\tau} & 0 & \frac{1}{\tau} & 0 \\
\frac{1}{\tau} & 0 & -3 \frac{1}{\tau} & 0 & \frac{1}{\tau} & \frac{1}{\tau} & 0 & 0 \\
\frac{1}{\tau} & 0 & 0 & -3 \frac{1}{\tau} & 0 & \frac{1}{\tau} & \frac{1}{\tau} & 0 \\
0 & \frac{1}{\tau} & \frac{1}{\tau} & 0 & -3 \frac{1}{\tau} & 0 & 0 & \frac{1}{\tau} \\
0 & 0 & \frac{1}{\tau} & \frac{1}{\tau} & 0 & -3 \frac{1}{\tau} & 0 & \frac{1}{\tau} \\
0 & \frac{1}{\tau} & 0 & \frac{1}{\tau} & 0 & 0 & -3 \frac{1}{\tau} & \frac{1}{\tau} \\
0 & 0 & 0 & 0 & \frac{1}{\tau} & \frac{1}{\tau} & \frac{1}{\tau} & -3 \frac{1}{\tau}
\end{array}\right]
$$

> eigenvals(A8);

$$
0,-6 \frac{1}{\tau^{\prime}}+4 \frac{1}{\tau} \cdot 2 \frac{1}{\tau^{\prime}}-4 \frac{1}{\tau^{\prime}}-2 \frac{1}{\tau} \cdot-4 \frac{1}{\tau} \cdot-2 \frac{1}{\tau}
$$

$>\mathrm{A} 8 \mathrm{R}:=\operatorname{matrix}([[1, \mathrm{~A}, \mathrm{~A}, \mathrm{~A}, \mathrm{~B}, \mathrm{~B}, \mathrm{~B}, \mathrm{C}],[\mathrm{A}, \mathbf{1}, \mathrm{B}, \mathrm{B}, \mathrm{A}, \mathrm{C}, \mathrm{A}, \mathrm{B}],[\mathrm{A}, \mathrm{B}, \mathbf{1}, \mathrm{B}, \mathrm{A}, \mathrm{A}, \mathrm{C}, \mathrm{B}],[\mathrm{A}, \mathrm{B}, \mathrm{B}, \mathbf{1}, \mathrm{C}, \mathrm{A}, \mathrm{A}, \mathrm{B}],[\mathrm{B}, \mathrm{A}, \mathrm{A}, \mathrm{C}, \mathbf{1}, \mathrm{B}, \mathrm{B}, \mathrm{A}],[\mathrm{B}, \mathrm{C}, \mathrm{A}$ $, A, B, 1, B, A],[B, A, C, A, B, B, 1, A],[C, B, B, B, A, A, A, 1]]) ;$

$$
A B R=\left[\begin{array}{llllllll}
1 & A & A & A & B & B & B & C \\
A & 1 & B & B & A & C & A & B \\
A & B & 1 & B & A & A & C & B \\
A & B & B & 1 & C & A & A & B \\
B & A & A & C & 1 & B & B & A \\
B & C & A & A & B & 1 & B & A \\
B & A & C & A & B & B & 1 & A \\
C & B & B & B & A & A & A & 1
\end{array}\right]
$$

$>$ eigenvals(A8R);

$3 B+1+3 A+C, 3 B+1-3 A-C, 1-B-A+C,-B+1+A-C, 1-B-A+C, B+1+A-C_{1} 1-B-A+C$.

avec $A=j_{0}(Q a) ; B=j_{0}(Q a \sqrt{2}) ; C=j_{0}(2 Q a)$ 


\section{Annexe 2}

\section{6 sites voisins sur un octaèdre $(1$ seul $\tau)$}

$>$ A6c:=matrix([[-4/tau, 1/tau,1/tau,1/tau,1/tau,0],[1/tau,-4/tau,1/tau,0,1/tau,1/tau],[1/tau,1/tau,4/tau,1/tau,0,1/tau],[1/tau,0,1/tau,-4/tau,1/tau,1/tau],[1/tau,1/tau,0,1/tau,4/tau,1/tau,$[0,1 /$ tau, $1 /$ tau, $1 /$ tau, $1 /$ tau,, $4 /$ tau $]]$;

$$
A G C=\left[\begin{array}{cccccc}
-4 \frac{1}{\tau} & \frac{1}{\tau} & \frac{1}{\tau} & \frac{1}{\tau} & \frac{1}{\tau} & 0 \\
\frac{1}{\tau} & -4 \frac{1}{\tau} & \frac{1}{\tau} & 0 & \frac{1}{\tau} & \frac{1}{\tau} \\
\frac{1}{\tau} & \frac{1}{\tau} & -4 \frac{1}{\tau} & \frac{1}{\tau} & 0 & \frac{1}{\tau} \\
\frac{1}{\tau} & 0 & \frac{1}{\tau} & -4 \frac{1}{\tau} & \frac{1}{\tau} & \frac{1}{\tau} \\
\frac{1}{\tau} & \frac{1}{\tau} & 0 & \frac{1}{\tau} & -4 \frac{1}{\tau} & \frac{1}{\tau} \\
0 & \frac{1}{\tau} & \frac{1}{\tau} & \frac{1}{\tau} & \frac{1}{\tau} & -\frac{1}{\tau}
\end{array}\right]
$$

$>$ eigenvals $(\mathbf{A} 6 \mathrm{c})$;

$$
0 .-6 \frac{1}{\tau} \cdot-6 \frac{1}{\tau},-4 \frac{1}{\tau} \cdot-4 \frac{1}{\tau},-4 \frac{1}{\tau}
$$

$>\mathrm{A} 6 \mathrm{cR}:=\operatorname{matrix}([[1, \mathrm{~A}, \mathrm{~A}, \mathrm{~A}, \mathrm{~A}, \mathrm{~B}],[\mathrm{A}, 1, \mathrm{~A}, \mathrm{~B}, \mathrm{~A}, \mathrm{~A}],[\mathrm{A}, \mathrm{A}, \mathrm{1}, \mathrm{A}, \mathrm{B}, \mathrm{A}],[\mathrm{A}, \mathrm{B}, \mathrm{A}, \mathrm{1}, \mathrm{A}, \mathrm{A}],[\mathrm{A}, \mathrm{A}$, $B, A, 1, A],[B, A, A, A, A, 1]]$;

$$
A G A R:=\left[\begin{array}{llllll}
1 & A & A & A & A & B \\
A & 1 & A & B & A & A \\
A & A & 1 & A & B & A \\
A & B & A & 1 & A & A \\
A & A & B & A & 1 & A \\
B & A & A & A & A & 1
\end{array}\right]
$$

$>$ eigenvals(A6cR);

$1+4 A+B 1+B-2 A 1+B-2 A, B-1-B, 1-B$

Avec $A=j_{0}(Q a \sqrt{2})$ et $B=j_{0}(2 Q a)$ 